\title{
NEW LOOK AT HIDDEN CHARM TETRA AND PENTAQUARK STATES***
}

\author{
Ahmed Ali $^{\mathrm{a}}$, Luciano Maiani ${ }^{\mathrm{b}}$, Anatoly V. Borisov ${ }^{\mathrm{c}}$ \\ Ishtiaq Ahmed $^{\mathrm{d}}$, M. JAmil Aslam ${ }^{\mathrm{e}}$ \\ Alexander Ya. Parkhomenko ${ }^{f}$, Antonio D. Polosa ${ }^{g}$ \\ ABDur Rehman ${ }^{d}$
}

${ }^{a}$ Deutsches Elektronen-Synchrotron DESY, 22607 Hamburg, Germany

${ }^{\mathrm{b}}$ Theory Division, CERN, Geneva, Switzerland

${ }^{c}$ Faculty of Physics, M.V. Lomonosov Moscow State University 119991 Moscow, Russia

${ }^{\mathrm{d}}$ National Centre for Physics, Quaid-i-Azam University Campus Islamabad 45320, Pakistan

ePhysics Department, Quaid-i-Azam University, Islamabad 45320, Pakistan

${ }^{\mathrm{f}}$ Department of Theoretical Physics, P.G. Demidov Yaroslavl State University Sovietskaya 14, 150003 Yaroslavl, Russia

'Dipartimento di Fisica and INFN, Sapienza Università di Roma

Piazzale Aldo Moro 2, 00185 Roma, Italy

\section{(Received April 24, 2018)}

The work presents the analysis of the hidden charm $P$-wave tetraquarks in the diquark model, using an effective Hamiltonian incorporating the dominant spin-spin, spin-orbit and tensor interactions. We compare them with other $P$-wave systems such as $P$-wave charmonia and the newly discovered $\Omega_{c}$ baryons, analyzed recently in this framework. The experimental situation on the $Y$-states is not certain, therefore, by using different spectra a detailed discussion of the parameters related to the diquark model is presented. Using the parameters from the currently preferred $Y$-states pattern, we work out the spin-parity, $J^{P C}$, and mass spectrum for many states in the hidden charm $P$-wave supermultiplet. We hope in future when there will be an experimental consensus on different $Y$-states, an observation of these new resonances would be a decisive footprint of the underlying diquark dynamics.

DOI:10.5506/APhysPolB.49.1315

* Presented by A. Rehman at the Cracow Epiphany Conference on Advances in Heavy Flavour Physics, Kraków, Poland, January 9-12, 2018.

** Based on the research performed in Ref. [1]. 


\section{Introduction}

The experimental discovery of the four-quark (more precisely, two quarks and two antiquarks) and five-quark (four quarks and an antiquark) states has opened new frontiers in QCD. The exotic four- and five-quark states, called $X, Y, Z$ and $P_{c}$, respectively, have been analyzed in a number of theoretical scenarios, reviewed recently in [2]. The objects of our interest in this work are the four $L=1$ states: $Y(4008), Y(4260), Y(4360)$, and $Y(4660)$ in the hidden charm $(c \bar{c})$ sector. While the last three have been measured in several experiments [3], the state $Y(4008)$ has been seen so far by Belle [4], about $250 \mathrm{MeV}$ below the well-established $Y(4260)$. There is preliminary support of this resonance from the BESIII data as well [5].

The $Y$-states have been interpreted as hadronic molecules [6], though $Y(4008)$ is not foreseen in this scenario. The state $Y(4260)$ has also been advocated as an example of a $(c \bar{c})_{8} g$ hybrid [7]. However, due to the recent evidence that $Y(4260)$ decays into $h_{c} \pi^{+} \pi^{-}[8]$ - a heavy-quark spin-flip transition - this interpretation is now disfavored. In the diquark-antidiquark model $[9,10]$, they are identified as $P$-wave $(L=1)$ states, with another foreseen with $L=3$ [11]. Tentatively, $Y(4360)$ and $Y(4660)$ were identified with the $n=2$ radial excitations of $Y(4008)$ and $Y(4260)$, respectively, due to their decays into $\psi(2 S) \pi^{+} \pi^{-}$, and the mass differences, which are similar to the ones, $\chi_{(c, b) J}(2 P)-\chi_{(c, b) J}(1 P)$, in the radial excitations of the quarkonium states [3]. It should be noted that in calculating the mass spectrum, tensor contribution was not included. We take a second look at the four $Y$-states in the diquark-antidiquark picture, treating them as $n=1 P$-wave states, with the mass differences accounted for by the spin-orbit, spin-spin and tensor contributions.

Closely related to the analysis of the $Y$-tetraquark states presented here there are the five narrow excited charmed-baryon states $\Omega_{c}(=c s s)$, whose mass spectrum has recently been measured by the LHCb Collaboration [12] and mainly confirmed by the Belle Collaboration [13]. The five $\Omega_{c}$-states are assumed to have the orbital angular momentum $L=1$ and negative parity. Their measured masses (in MeV) and suggested spins, $J$, are given in [12].

The $\Omega_{c^{-}}$-states and the doubly-charmed baryon $\Xi_{c c}^{++}$, which is found as a significant structure in the $\Lambda_{c}^{+} K^{-} \pi^{+} \pi^{+}$mass spectrum [14], can be analyzed in the same framework. We treat them as diquark-quark systems, $(c[s s])$, for the $\Omega_{c}$-baryons and $([c c] u)$ for the $\Xi_{c c}^{++}$-baryon, with the [ss]-diquark in the $\Omega_{c}$-state having the spin $S_{s s}=1$, and, likewise, for the [cc]-diquark in the $\Xi_{c c}^{++}$-baryon. With the $c$-quark spin being $S_{c}=1 / 2$ and $S_{s s}=1$, we have the total spin $S=1 / 2$ or $S=3 / 2$ for the $\Omega_{c}$-baryons. Combining the total spin with the orbital angular momentum $L=1$, yields the five observed $\Omega_{c}$-states, having $J=1 / 2$ (two states), $J=3 / 2$ (two states), and $J=5 / 2$ (one state). They have been discussed in a number of papers $[15-17,19]$. 
Here, we follow closely to the analysis by Karliner and Rosner [19], where the effects of the tensor contribution have been implemented in addition to the spin-spin and spin-orbit terms in calculating the mass spectrum.

For the $P$-wave tetraquark states in [11], the four basic $L=1$ resonances with $J^{P C}=1^{--}$of the diquark-antidiquark spectrum were identified with $Y(4008), Y(4260), Y(4290)$ (a broad structure in the $h_{c}$ channel), or $Y(4220)$ (a narrow structure in the same channel), and a possible $\Lambda \bar{\Lambda}$-resonance around $4630 \mathrm{MeV} . Y(4360)$ and $Y(4660)$, also known at that time, were tentatively considered to be the $n=2$ radial excitations of $Y(4008)$ and $Y(4260)$, respectively. Since that paper appeared, the experimental situation has evolved drastically, where the status of the $Y(4008)$ state is no longer established, and the state $Y(4260)$ is claimed by the BESIII Collaboration now as a double humped structure [5], which is resolved into two resonances: a lower component, $Y(4220)$, with observed decays into $h_{c} \pi^{+} \pi^{-}$and $\chi_{c 0} \omega$, and a higher component, $Y(4330)$, which decays into $J / \psi \pi^{+} \pi^{-}$. On the other hand, it was also observed that $Y(4630)$ and $Y(4660)$ could be fitted as a unique resonance, decaying mainly into the $\Lambda \bar{\Lambda}$-pair.

In conclusion, at present there seem to be two favored scenarios, SI and SII, both comprising of four $Y$-states and based essentially on the Belle, BaBar and BESIII data, namely:

- Scenario SI: $Y(4008), Y(4260), Y(4360), Y(4660)$, favored in [2];

- Scenario SII: $Y(4220), Y(4330), Y(4390), Y(4660)$, favored in [18].

In SI, $Y(4008)$ is assumed to be a real resonance and it features $Y(4260)$ as a single state. Belle [4] found that the data are better fitted with two resonances, $Y(4260)$ and $Y(4008)$, and the width of the $Y(4008)$ is found to be a factor 2 larger than that of $Y(4260)$. However, $Y(4008)$ has been seen so far by Belle only and current analysis from BESIII, concerning this resonance, is still inconclusive [5]. On this basis, SII discards $Y(4008)$ and, according to BESIII [5], features the two lines, $Y(4220)$ and $Y(4330)$, resolving $Y(4260)$, as the lowest resonances. $Y(4360)$ and $Y(4390)$ appearing in SI and SII, respectively, are considered as the same resonance seen in different experiments. Similarly, in both SI and SII, one considers $Y(4660)$ and the proposed $\Lambda \bar{\Lambda}$-resonance at $4630 \mathrm{MeV}$ to correspond to the same state. In order to justify the broad spectra of two scenarios that range over $400 \mathrm{MeV}$ in SI and $600 \mathrm{MeV}$ in SII, we refer to [1] where a detailed discussion is given.

The principal aim of the work presented here is to investigate whether the diquark picture provides a satisfactory description of the four $Y$-tetraquark states in the $c \bar{c}$ sector, and quantifies the strengths of the spin-spin, spin-orbit and tensor interactions, in addition to the diquark and charmed 
quark masses. In doing this, we first repeat the analysis of the five $L=1$ charmed baryons $\Omega_{c}$. While working in two scenarios, we expect that the value of the chromomagnetic, spin-spin coupling inside the diquark, $\left[\kappa_{c q}\right]_{P}$, to be close to the analogous parameter derived for the $S$-wave tetraquarks, which is $\left[\kappa_{c q}\right]_{S} \simeq 67 \mathrm{MeV}$ as obtained in [11]. For tight diquarks, this parameter should not be too much affected by the addition of one unit of the orbital angular momentum. In addition, comparison with the $S$-wave tetraquark masses can give the energy for the excitation of one unit of the orbital momentum. After the extraction of parameters is done, we obtain the predictions of the mass spectrum of full $P$-wave tetraquark multiplet. The details about the calculation of the tensor contribution into the orbitallyexcited tetraquark states can be found in Appendix A of [1]

\section{Tensor contribution}

In the diquark-quark picture, the effective Hamiltonian for the $\Omega_{c}$-states can be written as follows [19, 20]:

$$
\begin{aligned}
& H_{\mathrm{eff}}=m_{c}+m_{[s s]}+2 \kappa_{s s} \boldsymbol{S}_{s_{1}} \cdot \boldsymbol{S}_{s_{2}}+\frac{B_{\mathcal{Q}}}{2} \boldsymbol{L}^{2}+V_{\mathrm{SD}}, \\
& V_{\mathrm{SD}}=a_{1} \boldsymbol{L} \cdot \boldsymbol{S}_{s s}+a_{2} \boldsymbol{L} \cdot \boldsymbol{S}_{c}+b \frac{s_{12}}{4}+c \boldsymbol{S}_{s s} \cdot \boldsymbol{S}_{c} .
\end{aligned}
$$

Here, $m_{c}$ and $m_{[s s]}$ are the masses of the $c$-quark and [ss]-diquark, respectively, $\kappa_{s s}$ is the spin-spin coupling of the quarks in the diquark, and $\boldsymbol{L}$ is the orbital angular momentum of the diquark-quark system. The spindependent part $V_{\mathrm{SD}}(2)$ is taken from [19]. The coefficients $a_{1}$ and $a_{2}$ are the strengths of the spin-orbit terms involving the spin of the diquark $\boldsymbol{S}_{s s}$ and the charmed-quark spin $\boldsymbol{S}_{c}$, respectively. The term $b s_{12} / 4$ represents the tensor contribution (for details, see Eqs. (2)-(5) in [1])

$$
\frac{1}{2}\left\langle s_{12}\right\rangle=2\left\langle Q\left(\boldsymbol{S}_{s s}, \boldsymbol{S}_{c}\right)\right\rangle=\langle Q(\boldsymbol{S}, \boldsymbol{S})\rangle-\left\langle Q\left(\boldsymbol{S}_{c}, \boldsymbol{S}_{c}\right)\right\rangle-\left\langle Q\left(\boldsymbol{S}_{s s}, \boldsymbol{S}_{s s}\right)\right\rangle,
$$

where brackets mean an operator transformation to a space of states with a fixed orbital momentum and for $L=1$ (with $\boldsymbol{S}_{X}=\boldsymbol{S}, \boldsymbol{S}_{c}, \boldsymbol{S}_{s s}$ ), one gets [21]

$$
\left\langle Q\left(\boldsymbol{S}_{X}, \boldsymbol{S}_{X}\right)\right\rangle=-\frac{3}{5}\left[2\left\langle\left(\boldsymbol{L} \cdot \boldsymbol{S}_{X}\right)^{2}\right\rangle+\left\langle\boldsymbol{L} \cdot \boldsymbol{S}_{X}\right\rangle-\frac{4}{3}\left\langle\boldsymbol{S}_{X} \cdot \boldsymbol{S}_{X}\right\rangle\right] .
$$

In order to compute matrix elements of the operator $\boldsymbol{L} \cdot \boldsymbol{S}_{X}$, it is useful to construct the states with a definite $J_{3}$ as linear combinations of the states $\left|L_{3} ; S_{c 3}, S_{s s 3}\right\rangle$, where $S_{c 3}+S_{s s 3}+L_{3}=J_{3}$. For the recently observed five $\Omega_{c^{-}}$baryons [12] with the assumed assignment $J^{P}=1 / 2^{-}, 3 / 2^{-}, 5 / 2^{-}$, the above states are written in [19] (c.f. Eqs. (A7)-(A11)). However, there is a more efficient way to calculate the $\boldsymbol{L} \cdot \boldsymbol{S}_{X}$ matrix elements by using Wigner's 
$6 j$ symbols [21, 22] and its details are given in Appendix A of [1]. With both methods, the tensor contributions (3) to the $J^{P}=1 / 2^{-}, 3 / 2^{-}$, and $5 / 2^{-}$ states are as follows [1, 19]:

$$
\begin{aligned}
\frac{1}{4}\left\langle s_{12}\right\rangle_{J=1 / 2} & =\left(\begin{array}{cc}
0 & 1 / \sqrt{2} \\
1 / \sqrt{2} & -1
\end{array}\right), \\
\frac{1}{4}\left\langle s_{12}\right\rangle_{J=3 / 2} & =\left(\begin{array}{cc}
0 & -\sqrt{5} / 10 \\
-\sqrt{5} / 10 & 4 / 5
\end{array}\right), \quad \frac{1}{4}\left\langle s_{12}\right\rangle_{J=5 / 2}=-\frac{1}{5},
\end{aligned}
$$

where the appearance of non-diagonal elements in the matrices with $J^{P}=$ $1 / 2^{-}$and $3 / 2^{-}$is a direct indication of a state mixing [19]. After the effective Hamiltonian (1) is diagonalized, we separate the common mass term $M_{0}=$ $m_{c}+m_{[s s]}+\frac{\kappa_{s s}}{2}+B_{\mathcal{Q}}$ for all the five states, which is considered as a free parameter.

The parameters $a_{1}, a_{2}, b$, and $c$ in the spin-dependent term (2) are determined in [19] by using the spin-averaged mass and the mass differences of the five $\Omega_{c}$-states. We reproduce their values and summarize in Table I.

TABLE I

Values of the parameters $a_{1}, a_{2}, b, c$ from (2) and $M_{0}=m_{c}+m_{[s s]}+\frac{\kappa_{s s}}{2}+B_{\mathcal{Q}}$ (all in $\mathrm{MeV}$ ), determined from the $\Omega_{c}$-baryon mass spectrum [12].

\begin{tabular}{c|c|c|c|c}
\hline \hline$a_{1}$ & $a_{2}$ & $b$ & $c$ & $M_{0}$ \\
\hline 26.95 & 25.75 & 13.52 & 4.07 & 3079.94
\end{tabular}

The various parameters entering $M_{0}$ have been determined previously in the analysis of the tetraquark and pentaquark mass spectra, yielding $m_{c}=$ $1721 \mathrm{MeV}$ from the charmed baryons [23], $\kappa_{s s}=126 \mathrm{MeV}$ [24], and $m_{[s s]}=$ $740 \mathrm{MeV}$ [25]. This gives $B_{\mathcal{Q}} \simeq 556 \mathrm{MeV}$, from the value of $M_{0}$ quoted in Table I, which is somewhat higher than the value of $B_{\mathcal{Q}}=268 \mathrm{MeV}$ [11]. In future, if some $S$-wave states of $\Omega_{c}$-baryons are observed, a precise value of $B_{\mathcal{Q}}$ can be determined from the mass difference of the $P$ - and $S$-wave states.

Similar to the $\Omega_{c}$-baryons, the tensor contribution in $P$-wave tetraquark states (true neutral tetraquarks are of our interest only) can be incorporated by extending the Hamiltonian presented in [11]

$$
\begin{aligned}
H_{\mathrm{eff}}= & 2 m_{\mathcal{Q}}+\frac{B_{\mathcal{Q}}}{2} \boldsymbol{L}^{2}-3 \kappa_{c q}+2 a_{Y} \boldsymbol{L} \cdot \boldsymbol{S}+b_{Y} Q\left(\boldsymbol{S}_{\mathcal{Q}}, \boldsymbol{S}_{\overline{\mathcal{Q}}}\right) \\
& +\kappa_{c q}\left[2\left(\boldsymbol{S}_{q} \cdot \boldsymbol{S}_{c}\right)+2\left(\boldsymbol{S}_{\bar{q}} \cdot \boldsymbol{S}_{\bar{c}}\right)+3\right]
\end{aligned}
$$

Note that for true neutral tetraquarks and for the $Y$-states, in particular, with the quark content $[c q]_{3}[\bar{c} \bar{q}]_{3}$, where the subscripts denote the diquark 
and antidiquark color representations, the coefficients $a_{1 Y}$ and $a_{2 Y}$, being counterparts of $a_{1}$ and $a_{2}$ in (2) introduced for the $\Omega_{c}$-baryons, are the same due to the charged-conjugation invariance of the $Y$-states. Therefore, with the coefficient $a_{Y}=a_{1 Y}=a_{2 Y}$, there is one spin-orbit term, $a_{Y} \boldsymbol{L} \cdot \boldsymbol{S}$, only. The spin-spin interaction involving the $[c q]$-diquark $\boldsymbol{S}_{\mathcal{Q}}$ and $[\bar{c} \bar{q}]$-antidiquark $\boldsymbol{S}_{\overline{\mathcal{Q}}}$ spins is neglected [11], assuming the same suppression as for the $\Omega_{c}$-baryons (see Table I). The tensor contribution to the $P$-wave tetraquark masses can be again presented by using (4) as follows:

$$
\frac{1}{2}\left\langle s_{12}\right\rangle=2\left\langle Q\left(\boldsymbol{S}_{\mathcal{Q}}, \boldsymbol{S}_{\overline{\mathcal{Q}}}\right)\right\rangle=\langle Q(\boldsymbol{S}, \boldsymbol{S})\rangle-\left\langle Q\left(\boldsymbol{S}_{\mathcal{Q}}, \boldsymbol{S}_{\mathcal{Q}}\right)\right\rangle-\left\langle Q\left(\boldsymbol{S}_{\overline{\mathcal{Q}}}, \boldsymbol{S}_{\overline{\mathcal{Q}}}\right)\right\rangle .
$$

It should be noted that the non-vanishing tensor contribution comes from the $Y_{3^{-}}$and $Y_{4}$-states only, where spins of both the diquark and antidiquark are non-zero (see Eq. (12) of [1]). It can be written in the following matrix form:

$$
\frac{1}{4}\left\langle s_{12}\right\rangle=\left(\begin{array}{cc}
0 & 2 / \sqrt{5} \\
2 / \sqrt{5} & -7 / 5
\end{array}\right),
$$

where the off-diagonal entries are due to the mixing in the $Y_{3}-Y_{4}$ system. Using Eq. (6), we get the masses of the four $Y$-tetraquarks

$$
\begin{array}{ll}
M_{1}=M\left(Y_{1}\right)=M_{00}-3 \kappa_{c q} \equiv \widetilde{M}_{00}, & M_{2}=M\left(Y_{2}\right)=\widetilde{M}_{00}-2 a_{Y}+2 \kappa_{c q}, \\
M_{3}=\widetilde{M}_{00}+4 \kappa_{c q}+E_{+}, & M_{4}=\widetilde{M}_{00}+4 \kappa_{c q}+E_{-},
\end{array}
$$

where $E_{ \pm}=\left[-30 a_{Y}-7 b_{Y} \mp \sqrt{3} \sqrt{300 a_{Y}^{2}+140 a_{Y} b_{Y}+43 b_{Y}^{2}}\right] / 10$ are the eigenvalues of the matrix $2 a_{Y}\langle\boldsymbol{L} \cdot \boldsymbol{S}\rangle+b_{Y}\left\langle s_{12}\right\rangle / 4$ and $M_{00}=2 m_{\mathcal{Q}}+B_{\mathcal{Q}}$. There are the relations among $M_{3}$ and $M_{4}$

$$
\begin{aligned}
M_{3}+M_{4} & =2\left(\widetilde{M}_{00}+4 \kappa_{c q}\right)-\frac{1}{5}\left(30 a_{Y}+7 b_{Y}\right) \\
& =2\left(\widetilde{M}_{00}+4 \kappa_{c q}\right)+E_{+}+E_{-}, \\
M_{4}-M_{3} & =\frac{\sqrt{3}}{5} \sqrt{300 a_{Y}^{2}+140 a_{Y} b_{Y}+43 b_{Y}^{2}}=E_{-}-E_{+} .
\end{aligned}
$$

The diquark-diquark-antiquark approach has been applied for the calculation of the mass spectrum of the hidden-charm pentaquarks recently [24]. Tensor contributions in pentaquark states are in progress [26].

\section{Numerical analysis}

The parameters in the analysis of the $Y$-states are sensitive to the input values of their masses. Currently, there is no consensus on them, and we have 
two different scenarios. In the first scenario (SI), the masses (all in $\mathrm{MeV}$ ) of the four $J^{P C}=1^{--} Y$-states, known as $Y(4008), Y(4260), Y(4360)$, and $Y(4660)$, are as follows:

$$
\begin{array}{ll}
M_{1}=4008 \pm 40_{-28}^{+114}, & M_{2}=4230 \pm 8, \\
M_{3}=4341 \pm 8, & M_{4}=4643 \pm 9 .
\end{array}
$$

Here, all the masses are taken from the PDG review [3], except for $Y(4008)$, which is from the Belle paper [4]. In the Belle analysis, it was found that the data are better fitted with two resonances, $Y(4260)$ and $Y(4008)$, and the width of the state $Y(4008)$ is found to be a factor 2 larger than that of $Y(4260)$.

The second scenario (SII) is, in general, supported by the BESIII Collaboration [27]. Discarding their $Y$-state with the mass $3846 \pm 45.5 \mathrm{MeV}$, and assigning $Y(4220), Y(4320)$, and $Y(4390)$ with $Y_{1}, Y_{2}$, and $Y_{3}$, respectively, their masses are (all in $\mathrm{MeV})$ :

$$
\begin{aligned}
& M_{1}=4219.6 \pm 3.3 \pm 5.1, \quad M_{2}=4333.2 \pm 19.9 ， \\
& M_{3}=4391.5 \pm 6.3, \quad M_{4}=4643 \pm 9 .
\end{aligned}
$$

The state $Y_{4}$ in Eqs. (11) and (12) is the same but the others differ significantly.

Before proceeding to the estimate of the parameters $M_{00}, a_{Y}, \kappa_{q c}$, and $b_{Y}$, we first specify their possible interdependence on each other. From $M_{4}-M_{3}$ (10) follows that this mass difference is invariant under the simultaneous sign change $\left(a_{Y}, b_{Y}\right) \rightarrow\left(-a_{Y},-b_{Y}\right)$. Hence, from this mass difference alone, we have two solutions, and we discard those which give $a_{Y}<0$, in line with the analysis for the $\Omega_{c^{-}}$-baryons, given in Table I. This is also the choice suggested by the mass ordering, in which the $L=3$ state, called $Y_{5}$, should have a higher mass than all the $L=1$ states. So, the only physically acceptable solution is the one which has the positive value of $a_{Y}$ irrespectable to the sign of the $b_{Y}$ value. In addition to Eq. (10), the mass difference $M_{2}-M_{1}$ provides a constraint on the parameters $a_{Y}$ and $\kappa_{c q}$

$$
M_{2}-M_{1}=2\left(\kappa_{c q}-a_{Y}\right)>0 .
$$

Thus, in both the scenarios for the $Y$-tetraquark masses, $\kappa_{c q}>a_{Y}$, with the two approaching each other as this mass difference decreases. The central values of the parameters $a_{Y}, b_{Y}, \kappa_{c q}$, and $M_{00}$ are determined from the masses given in Eqs. (11) and (12) and presented in Table II.

Recalling the definition, $M_{00}=2 m_{\mathcal{Q}}+B_{\mathcal{Q}}$, the mass of the diquark, $m_{\mathcal{Q}}=m_{[c q]}$, comes out to be 2108 (1964) $\mathrm{MeV}$ and 2133.36 (1989.36) MeV, in the case 2 of Scenarios I and II, respectively, corresponding to $B_{\mathcal{Q}}=$ 
Values (all in MeV) of the parameters in Scenarios I (SI) and II (SII). Here, c1 and $\mathrm{c} 2$ abbreviate the cases 1 and 2, respectively.

\begin{tabular}{l|r|r|r|r}
\hline \hline & \multicolumn{1}{|c|}{$a_{Y}$} & \multicolumn{1}{c|}{$b_{Y}$} & \multicolumn{1}{c|}{$\kappa_{c q}$} & \multicolumn{1}{c}{$M_{00}$} \\
\hline SI (c1) & -22.11 & -88.73 & 88.88 & 4274.66 \\
SI (c2) & 47.67 & 10.95 & 158.67 & 4484.01 \\
SII (c1) & -3.22 & -105.25 & 53.57 & 4380.31 \\
SII (c2) & 48.23 & -31.73 & 105.03 & 4534.72
\end{tabular}

268 (556) $\mathrm{MeV}$, extracted from the earlier analysis of the tetraquark states (and from the $\Omega_{c}$-baryons). We have repeated this numerical analysis by taking into account the errors on the $M_{i}(i=1, \ldots, 4)$ masses, shown above. In doing this, we have added the errors in quadrature, and as the errors are highly asymmetric (in $M_{1}$ in Scenario I), we have averaged them. The resulting errors on the parameters $a_{Y}, b_{Y}$, and $\kappa_{c q}$ are shown in Table III, together with their central values. This exercise underscores that the tensor coupling $b_{Y}$ has a large uncertainty due to the current dispersion in the $Y$-tetraquark masses.

TABLE III

The values of the parameters $a_{Y}, b_{Y}$, and $\kappa_{c q}$ with errors in Scenarios I (SI) and II (SII) (all in $\mathrm{MeV}$ ).

\begin{tabular}{l|c|r|c}
\hline \hline & $a_{Y}$ & \multicolumn{1}{|c|}{$b_{Y}$} & \multicolumn{1}{c}{$\kappa_{c q}$} \\
\hline SI (c1) & $-22.11 \pm 29.40$ & $-88.73 \pm 68.46$ & $88.88 \pm 9.01$ \\
SI (c2) & $47.67 \pm 20$ & $10.95 \pm 81.88$ & $158.67 \pm 18.41$ \\
SII (c1) & $-3.22 \pm 12.33$ & $-105.25 \pm 19.85$ & $53.57 \pm 5.42$ \\
SII (c2) & $48.23 \pm 4.42$ & $-31.73 \pm 31.14$ & $105.03 \pm 2.49$
\end{tabular}

Before concluding, we give the mass formula for the $Y_{5}$-state with $L=3$

$$
M\left(Y_{5}\right)=2 m_{\mathcal{Q}}+6 B_{\mathcal{Q}}-16 a_{Y}+\kappa_{c q}-\frac{8}{5} b_{Y}
$$

Without the $b_{Y}$ term, it has been derived in [11], albeit with the opposite sign of $\kappa_{c q}$. Using the values of parameters given in Table II along with the value of $m_{\mathcal{Q}}$ calculated for $B_{\mathcal{Q}}=268(556) \mathrm{MeV}$, the predicted masses of the $Y_{5}$-state in the case 2 of SI and SII are 5202 (6642) MeV and 5259 (6699) $\mathrm{MeV}$, respectively. A precise value of $B_{\mathcal{Q}}$ is required to reduce the current uncertainty in $M_{5}$. We expect many other particles in the supermultiplet of the $P$-wave diquarkonia, analogous to the $\chi$-states of charmonia 
and bottomonia. Their quantum numbers are as follows (in parenthesis, the multiplicity of each state is given):

$$
\begin{aligned}
& 3^{--}(1) ; \quad 2^{--}(2) ; \quad 2^{-+}(2) ; \\
& 1^{--}(4) ; \quad 1^{-+}(2) ; \quad 0^{--}(1) ; \quad 0^{-+}(2) \text {. }
\end{aligned}
$$

The total number of states coincides with the total number of quark spin and orbital momentum states, i.e., $2^{4} \times 3=48$, as one can verify easily. The tentative masses of these states lie in the range from 4250 to $4670 \mathrm{MeV}$ and can be found in Table 6 of Ref. [1].

Indications exist for two $0^{-+}$states. However, in the same channel, there should appear two conventional radially excited charmonia, $\eta_{c}(3 S)$ and $\eta_{c}(4 S)$, for a total of four states, with possible mixing and corresponding distortions of the spectrum.

\section{Conclusions and outlook}

We have derived the tensor contribution to the masses of the $P$-state tetraquarks using the effective Hamiltonian in the diquark-antidiquark picture, correlating the Hamiltonian parameters with those determined from the analysis of the five excited $\Omega_{c}$-baryons in the same approach. We find that whereas the other parameters are in the right ball-park, the coupling $b_{Y}$ remains essentially undetermined due to the current uncertainties in the masses of $Y$-tetraquarks. Hence, there is an urgent need to revisit these states experimentally, which hopefully can be done by the BESIII, Belle II, and $\mathrm{LHCb}$ collaborations. With precise measurements done, the parameters of the effective Hamiltonian can be determined more accurately, providing a quantitative test of the underlying diquark model.

\section{REFERENCES}

[1] A. Ali et al., Eur. Phys. J. C 78, 29 (2018) [arXiv:1708.04650 [hep-ph]].

[2] A. Ali, J.S. Lange, S. Stone, Prog. Part. Nucl. Phys. 97, 123 (2017) [arXiv:1706.00610 [hep-ph]].

[3] C. Patrignani et al. [Particle Data Group], Chin. Phys. C 40, 100001 (2016).

[4] K. Abe et al. [Belle Collaboration], arXiv:hep-ex/0612006.

[5] M. Ablikim et al. [BESIII Collaboration], Phys. Rev. Lett. 118, 092001 (2017) [arXiv:1611.01317 [hep-ex]].

[6] Q. Wang, C. Hanhart, Q. Zhao, Phys. Rev. Lett. 111, 132003 (2013) [arXiv:1303.6355 [hep-ph]].

[7] F.E. Close, P.R. Page, Phys. Lett. B 628, 215 (2005) [arXiv:hep-ph/0507199]. 
[8] M. Ablikim et al. [BESIII Collaboration], Phys. Rev. Lett. 118, 092002 (2017) [arXiv:1610.07044 [hep-ex]].

[9] R.L. Jaffe, Phys. Rev. D 15, 267 (1977).

[10] R.L. Jaffe, F. Wilczek, Phys. Rev. Lett. 91, 232003 (2003) [arXiv:hep-ph/0307341].

[11] L. Maiani, F. Piccinini, A.D. Polosa, V. Riquer, Phys. Rev. D 89, 114010 (2014) [arXiv:1405.1551 [hep-ph]].

[12] R. Aaij et al. [LHCb Collaboration], Phys. Rev. Lett. 118, 182001 (2017) [arXiv:1703.04639 [hep-ex]].

[13] J. Yelton et al. [Belle Collaboration], Phys. Rev. D 97, 051102 (2018) [arXiv:1711.07927 [hep-ex]].

[14] R. Aaij et al. [LHCb Collaboration], Phys. Rev. Lett. 119, 112001 (2017) [arXiv:1707.01621 [hep-ex]].

[15] W. Wang, R.L. Zhu, Phys. Rev. D 96, 014024 (2017) [arXiv:1704.00179 [hep-ph]].

[16] M. Padmanath, N. Mathur, Phys. Rev. Lett. 119, 042001 (2017) [arXiv: 1704.00259 [hep-ph]].

[17] T.M. Aliev, S. Bilmis, M. Savci, arXiv:1704.03439 [hep-ph].

[18] S.L. Olsen, T. Skwarnicki, D. Zieminska, Rev. Mod. Phys. 90, 015003 (2018) [arXiv:1708.04012 [hep-ph]].

[19] M. Karliner, J.L. Rosner, Phys. Rev. D 95, 114012 (2017) [arXiv:1703.07774 [hep-ph]].

[20] M. Karliner, J.L. Rosner, Phys. Rev. D 92, 074026 (2015) [arXiv:1506.01702 [hep-ph]].

[21] L.D. Landau, E.M. Lifshitz, Quantum Mechanics (Nonrelativistic Theory), Oxford: Pergamon Press, 1977, p. 96.

[22] A.R. Edmonds, Angular Momentum in Quantum Mechanics, Princeton: Princeton University Press, 1957.

[23] L. Maiani, F. Piccinini, A.D. Polosa, V. Riquer, Phys. Rev. D 71, 014028 (2005) [arXiv:hep-ph/0412098].

[24] A. Ali, I. Ahmed, M.J. Aslam, A. Rehman, Phys. Rev. D 94, 054001 (2016) [arXiv: 1607.00987 [hep-ph]].

[25] N.V. Drenska, R. Faccini, A.D. Polosa, Phys. Lett. B 669, 160 (2008) [arXiv:0807.0593 [hep-ph]].

[26] A. Ali et al., in preparation.

[27] X.Y. Gao, C.P. Shen, C.Z. Yuan, Phys. Rev. D 95, 092007 (2017) [arXiv:1703.10351 [hep-ex]]. 